\title{
OFF-ROAD AND ON-ROAD DRIVING ASSESSMENTS METHODS, WHAT DO THEY SAY? A CLINICAL SAMPLE
}

\author{
Helena Larsson, ${ }^{1}$ Torbjörn Falkmer ${ }^{2}$ \\ ${ }^{1}$ NVS department, Clinical Geriatrics \\ Karolinska Institutet - Huddinge \\ Stockholm, Sweden. \\ ${ }^{2}$ Rehabilitation Medicine \\ Department of Neuroscience and Locomotion, INR \\ Faculty of Health Sciences \\ Linköping University, Sweden \\ School of Health Sciences, HHJ \\ Jönköping University, Sweden \\ E-mail: helena.u.larsson@ki.se, torbjorn.falkmer@hjj.hj.se
}

\begin{abstract}
Summary: Driving assessments are carried out in various ways, and diverse methods and tests are used. The British Stroke Driver Screening Assessment (SDSA) is a cognitive test, developed to evaluate driving fitness in stroke patients. For Sweden, Denmark and Norway, there is a Nordic version, the Nordic Stroke Driver Screening Assessment (NorSDSA). This cognitive test has become commonly used by driving assessors and is sometimes the only test used to evaluate driving fitness regardless of a client's diagnoses. The objective of the study was thus to compare the off-road NorSDSA test outcome, expressed as a global pass or fail result, with an on-road driving test outcome. The results showed that 52 out of 63 patients were correctly classified in the drivers-fail group and 24 of 57 in the pass group. In total, 63\% were correctly classified, (sensitivity $42 \%$ and specificity $83 \%$ ). The NorSDSA is thus not a valid predictor for pass or fail on the on-road test. However, only a few neuropsychological tests are made for driving assessments. The NorSDSA is one of them, but driving assessors should know its apparent limitations. The recommendation is thus not to solely rely on the NorSDSA to assess fitness to drive.
\end{abstract}

\section{BACKGROUND}

Driving is an instrumental activity of daily living (IADL) and plays an important role in modern society (Liddle \& McKenna, 2003; Pedretti, 1996). Even when other forms of transportation are available, driving often remains the most satisfying and preferred travel mode (Gillins, 1990), and is in fact, taken for granted by most people. However, compared to other skills, driving is a very complex activity, which cannot be reduced to the mere technical operation of a vehicle, and can therefore never be completely routine (Fox, Bowden, \& Smith, 1998). Medical conditions, for example following a disease or trauma, often call into question a person's fitness to drive, but the diagnosis alone cannot predict the driving ability.

A vast majority of all diagnoses relevant to fitness-to-drive status affect older drivers; hence, they constitute the group of interest (Breker et al., 2003; Hakamies-Blomqvist, Sirén, \& Davidse, 2003). Older drivers have no particular problem with automatic processes, which place limited 
demands on their attentional capacity and generally occur under highly predictable conditions of traffic and weather (Breker et al., 2003). Non-automatic processes, such as those used for unpredictable or unfamiliar demands like turning, merging, collision avoidance or driving in adverse weather, are more challenging for older drivers (Breker et al., 2003; HakamiesBlomqvist, Sirén, \& Davidse, 2003; Maycock, 1996). Off-road tests should, preferably, detect these problems that may occur in the target group. Neuropsychological tests, simulators and/or on-road tests are often used for this reason to assess cognitive function and driving performance.

\section{Off-road tests}

The British Stroke Driver Screening Assessment (SDSA) is a cognitive test developed to evaluate driving fitness in stroke patients (Nouri \& Lincoln, 1992). The SDSA (and NorSDSA) comprises the four following tests, yielding six measurements:

- Dot cancellation

- Square matrices with the

- direction test

- compass test

- $\quad$ Road sign recognition

$-3 \mathrm{~min}$

$-5 \mathrm{~min}$

To predict fitness to drive, four of these scores, namely Dot cancellation (time and score), Square matrices: compass test, Road sign recognition $-3 \mathrm{~min}$, are entered into an equation derived from discriminant function analysis. In a study by Radford and Lincoln (2004), SDSA was shown to be sensitive to impairment in executive abilities and attention. From an experimental study, SDSA correctly predicted the on-road test performance of $81 \%$ of stroke patients, sensitivity 75\% and specificity 89\% (Nouri \& Lincoln, 1993). A test/retest-reliability has also been investigated (Lincoln \& Fanthome, 1994). Twenty-six stroke patients were tested on two occasions six weeks apart. There were some improvements on Dot cancellation and Road sign recognition but no patient improved from a "fail" to a "pass." For Sweden, Denmark and Norway, there is a Nordic version, called the Nordic Stroke Driver Screening Assessment: NorSDSA (Lundberg, Caneman, Samuelsson, Hakamies-Blomqvist, \& Almkvist, 2003). Some adaptations were made on the Nordic version; e.g., the cards for the Square matrices regarding the position of the driver (for right-hand traffic) and other road-signs for the Road sign recognition test. In addition, the NorSDSA has a cut-off limit of 0.5 instead of 0 as in SDSA.

\section{On-road test}

Although on-road testing is often seen as the gold standard, the outcome is nevertheless subjective, based on each evaluator's judgment. On-road tests measure both the operational (maneuvering) and cognitive aspects of driving and should be assessed with a special observational scoring sheet. Different protocols have been developed to obtain patients' actual driving performance (Akinwuntan et al., 2003; Hunt et al., 1997; Odenheimer et al., 1994; Patomella, Tham, \& Kottorp, 2006). However, depending on the background of the assessor (e.g., driving instructor, occupational therapist, etc.) and which assessment protocol being used, 
the on-road test's objectivity and validity may be questioned, which, in turn, implies low reliability.

Many studies have been published on the determination of the driving potential for different diagnostic groups (for example: Hunt, 1993; Lundqvist et al., 1997; McKenna, Jefferies, Dobson, \& Frude, 2004; Schanke \& Sundet, 2000). The focus has been on correlations between neuro psychological tests and on-road test outcomes, but few of these neuro psychological tests are developed specially for driving. In clinical practice, it is not rare to believe that neuro psychological tests could be a valid predictor for fitness to drive (Larsson, Lundberg, Falkmer, \& Johansson, 2007). In Sweden, the NorSDSA has become more commonly used and is sometimes the only test to evaluate driving fitness, regardless of the clients' diagnoses (Larsson et al., 2007), an approach that may be questioned. The objective of the present study was thus to compare the off-road NorSDSA test outcome, expressed as a global pass or fail result and as sub score results, with a on-road driving test outcome for clients with different diagnoses, as well as different ages, education and sex.

The following research questions were posed:

1. Is the NorSDSA a valid screening instrument for the outcome of on-road testing?

2. Does the age of a client have an impact on the outcome of the on-road test?

3. Does the sex of a client have an impact on the outcome of the on-road test?

4. Does the educational level of a client have an impact on the outcome of the on-road test?

5. Does the educational level of a client have an impact on the outcome of the NorSDSA?

6. Do any of the subscales in NorSDSA have a better predictive value on the outcome of onroad test?

7. Does the diagnosis of a client have an impact on the outcome of the on-road test?

8. Does the diagnosis of a client have an impact on the outcome of the NorSDSA?

\section{METHOD}

In total, 120 patients (100 men and 20 women) ranging from 43-88 years old (mean age $=68.6$, S.D. $=10.3$ ) were selected from a database at a driving assessment unit in Sweden. This group consisted of 57 patients with CVI, 47 patients with cognitive deficits, 6 patients with traumatic brain injury TBI and 10 unspecified disorders (other). All patients had been assessed during the course of normal clinical evaluation of fitness to drive, which also included NorSDSA and a onroad driving test (the inclusion criteria was results from both the NorSDSA and on-road test). All patients drove cars with manual or automatic gear shifts, equipped with a dual-brake system, on the same standard route for about 60 minutes. The route included a diversity of road types; suburban streets, motorways and country roads. The on-road driving tests were assessed by an experienced occupational therapist who scored their performance with P-Drive; Performance Analysis of Driving Ability (Patomella, Tham \& Kottorp, 2006), but the final outcome of the driving test (pass/fail) was the result of a global impression of the patient's performance. A driving instructor was responsible for specific instructions (directions on the route) and security (dual controls). 


\section{Statistical analyses}

Statistical analyses were carried out with $\operatorname{SPSS}^{\circledR}$ (14.0 version). $\chi^{2}$ tests and Kruskal Wallis test were used for non-parametric analyses of ordinal and categorical data. Student's t test for independent samples and one-way analyses of variance (ANOVA) with post hoc test Tukey HSD were used for analyses on parametric data. The $\alpha$-level was set to .05 for all statistical tests.

\section{RESULTS}

As shown in Table 1, of 120 patients, 57 (48\%) passed the on-road test, while $63(52 \%)$ failed the on-road test.

Table 1. The NorSDSA for prediction on pass/fail on the on-road test

\begin{tabular}{lccc} 
& Fail on-road test & Pass on-road test & Total \\
\hline Fail NorSDSA & 52 & 33 & 85 \\
Pass NorSDSA & 11 & 24 & 35 \\
\hline Total & 63 & 57 & 120
\end{tabular}

Hence, the sensitivity of the NorSDSA was $42 \%$ and the specificity $83 \%$ on the outcome of the on-road test. The positive predictive value was $(24 / 35=69 \%)$, and the negative predictive value was $(52 / 85=61 \%)$. The overall screening score was 125 , i.e., $63 \%$.

Each patient was then assigned to an on-road test pass $(n=57)$ or a fail $(n=63)$ group. Subjects in the on-road test fail-group were older than patients in the pass-group; 72 years (SD 8.9) vs. 65 years (SD 10.5), $(\mathrm{p}<.001)$. There were no differences between men and women on the on-road test outcome. Years of education were clustered into two categories: university studies (13+ years) or not ( $<13$ years). The educational level did not have any impact on the outcome of the on-road test. Furthermore, no significant differences with respect to years in school, neither on the over all score for NorSDSA, nor on any of the sub scores, were found.

The on-road test pass and fail groups were compared with respect to the outcome of the sub scores of NorSDSA. The two groups showed significantly different outcomes on the NorSDSA direction test, compass test and road sign recognition $3 \mathrm{~min}$ and $5 \mathrm{~min}$ tests (all pass group's tests scores being better, $\mathrm{p}<.01$ for each test).

Secondly, the patients were divided into four groups depending on their diagnoses; cognitive deficits $(n=47)$, CVI $(n=57)$, TBI $(n=6)$, and other $(n=10)$. The diagnosis itself did not affect the on-road test outcome. The NorSDSA test and sub tests were run through an ANOVA with post hoc testing to find out if the outcome of NorSDSA varied between the different diagnoses. The clients with cognitive deficits scored significantly worse on the overall NorSDSA score $(\mathrm{p}<.05)$, and on the sub score compass test $(\mathrm{p}<.05)$, and road sign recognition 5 min test $(\mathrm{p}<.01)$ compared to the group of subjects with TBI. The same was true for clients with cognitive deficits, who scored significantly worse compared to the CVI group (compass test, $\mathrm{p}<.05$, and road sign recognition 5 min test, $\mathrm{p}<.01$ ), except for the overall score on NorSDSA, in which no significant differences were found between clients with cognitive deficits and the CVI group. No 
other diagnoses scored differently from each other, neither on any of the sub tests, nor on the overall NorSDSA test.

In order to check whether age might be a confounding factor, the diagnosis groups were compared in this respect. Significant differences were found, as shown in Table 2.

Table 2. Age within the different diagnosis groups

\begin{tabular}{lllll} 
Diagnosis group & $\mathrm{n}$ & Mean age & SD & $\mathrm{p}$ (cognitive deficits vs. all other groups) \\
\hline Cognitive deficits & 47 & 73.2 & 8.0 & \\
CVI & 57 & 66.7 & 10.5 & $\mathrm{p}<.01$ \\
TBI & 6 & 61.4 & 13.1 & $\mathrm{p}<.05$ \\
Other & 10 & 62.2 & 9.2 & $\mathrm{p}<.01$ \\
\hline Overall & & 68.6 & 10.3 & $\mathrm{p}<.01$
\end{tabular}

\section{DISCUSSION}

The NorSDSA was not a valid predictor for pass or fail on the on-road test. Despite a specificity of $83 \%$ for the Nor SDSA in predicting the outcome of on-road testing, the screening score of 125 and a sensitivity actually lower than that of tossing a coin tell us that the NorSDSA should not be used alone to predict driving ability in the diagnosis groups used for this study. This finding should, however, be taken with some care, since it may be due to several reasons. Firstly, the outcome variable, the on-road test, may suffer from too many unpredictable events triggering different task demands on the person tested. The on-road test suffers from low reliability, as well as, potentially, a lack of validity (Goldenbeld, Baughan, \& Hatakka, 1999). However, utilizing an on-road test often makes it possible to determine whether cognitive impairments found by the NorSDSA may be compensated for by the satisfactory driving skills of (usually) experienced drivers. On-road tests have a strong face validity but may very well lack construct validity. From this point of view, it is puzzling that on-road testing is viewed as the golden standard, but most likely this is due to the simple fact that there are no alternatives. Hence, further research on this topic is needed.

Secondly, there are also differences in the previous SDSA-studies regarding the cars used in the driving tests. Often a dual-control car has been used, but in the study by Lundberg et al. (2003), the majority of the patients drove their own cars. In the present study, all patients drove a car with dual controls.

Lastly, this particular cohort of subjects - half of whom failed the on-road test — could be a very special sub sample of subjects, largely deviating from the population the test was originally constructed for. The SDSA was designed and validated for stroke patients and later on for TBI patients (Radford \& Lincoln, 2004; Radford, Lincoln, \& Murray-Leslie, 2004), but in Sweden it is often used clinically to assess different and mixed diagnostic groups, as in this study (Larsson et al., 2007). Another limitation in the present study is the relatively small number of subjects in each diagnostic group, especially the TBI and Other groups. Yet another drawback is that we do not know the actual impact of this confounding variable on the outcome of the on-road test. However, the diagnosis itself did not affect the on-road test outcome, which may be due to the fact that either the test is sensitive to all included diagnoses, or more likely, that too few subjects 
in at least two of the diagnosis groups together with a fair specificity did catch also patients actually fit to drive in all groups. The reason for this statement is that the apparent lack of sensitivity does not speak in favor of the former argument.

The age of the client had a great impact on the outcome of the on-road test. However, the different diagnosis groups also turned out to differ significantly with respect to age. This means that we cannot draw any conclusion from the analysis on the impact of the different diagnoses with respect to the outcome of the NorSDSA, since it could very well reflect the age of the client, as well. Interestingly, males and females did not perform differently on the on-road test. In general, men drive on average far more miles per year than women (Evans, 2005), and apparently_-with this sample of clients - experience did not have an impact on the on-road test. However, neither we do not know the physical status of the males vs. females, nor do we know their actual yearly mileage, which, of course, could be further confounding factors. In addition, only 20 women participated in the study.

The educational level had no impact on the outcome of the on-road test or on the entire NorSDSA battery. This finding was somewhat surprising since generally, educational level is reflected when it comes to crash involvement in traffic (Evans, 2005).

The patients that passed the on-road test scored better on four of the sub scores (i.e., the direction test, compass test and road sign recognition 3 min and 5 min tests) than those who failed. This finding advocates that some of the subtests might perform better in a screening score test than the entire battery. However, to pick just one subtest out of a test battery is a dubious strategy, removing any validity and reliability adhered to the test.

Some of the diagnosis groups performed significantly better than others on the different sub tests within the Nor SDSA, but in light of the finding that the patient's diagnosis was not reflected by the outcome of the on-road test, this finding should not be disregarded.

\section{CONCLUSIONS}

Using the NorSDSA to assess fitness to drive based on on-road testing showed a screening score of 125 (i.e., 63\%), which is slightly better than tossing a coin. The NorSDSA is thus not a valid predictor for pass or fail on the on-road test. However, only a few neuropsychological tests are designed for driving assessments. The NorSDSA is one of them, but driving assessors should be aware of its apparent limitations. The recommendation is thus not to solely rely on the NorSDSA to assess fitness to drive.

\section{REFERENCES}

Akinwuntan, A.E., DeWeerdt, W., Feys, H., Baten, G., Arno, P., \& Kiekens, C. (2003). Reliability of a road test after stroke. Arch Phys Med Rehabil, 84, 1792-1796.

Breker, S., Henrikson, P., Falkmer, T., Bekiaris, E., Panou, M., Eeckhout, G., et al. (2003). Problems of elderly in relation to the driving task and relevant critical scenarios (Deliverable report No. D.1.1.): AGILE - AGed people Integration, mobility, safety and 
quality of Life Enhancement through driving. EU project - part of the Quality of Life and Management of Living Resources, Contract N. QLRT-2001-00118.

Fox, G.K., Bowden, S.C., \& Smith, D.S. (1998). On-road assessment of driving competence after brain impairment: review of current practice and recommendations for a standardized examination. Arch Phys Med Rehabil, 79(10), 1288-1296.

Gillins, L. (1990). Yielding to age: when the elderly can no longer drive. Journal of Gerontological Nursing, 16(11), 12-15.

Evans, L. (2005). Traffic Safety (1 ed.). Bloomfield Hills: Science Serving Society.

Goldenbeld, C., Baughan, C.J., \& Hatakka, M. (1999). Driver testing. In S. Siegrist (Ed.), Driver Training, Testing and Licensing - towards theory-based management of young driver's injury risk in road traffic. Berne: Schoch+Co.AG, 257.

Hakamies-Blomqvist, L., Sirén, A., \& Davidse, R. (2003). Older Drivers - a Review (VTI Report 497A). Linköping: VTI.

Hunt, L.A. (1993). Evaluation and retraining programs for older drivers. Clin Geriatr Med, 9(2), 439-448.

Hunt, L.A., Murphy, C.F., Carr, D., Duchek, J.M., Buckles, V., \& Morris, J.C. (1997). Reliability of the Washington University Road Test. A performance-based assessment for drivers with dementia of the Alzheimer type. Arch Neurol, 54(6), 707-712.

Larsson, H., Lundberg, C., Falkmer, T., \& Johansson, K. (In press). A Swedish Survey of Occupational Therapist's Involvement and Performance in Driving Assessments. Scand J Occup Ther.

Liddle, J., \& McKenna, K. (2003). Older drivers and driving cessation. British Journal of Occupational Therapy, 66(3), 125-132.

Lincoln, N.B., \& Fanthome, Y. (1994). Reliability of the Stroke Drivers Screening Assessment. Clinical Rehabilitation, 8(2), 157-160.

Lundberg, C., Caneman, G., Samuelsson, S.M., Hakamies-Blomqvist, L., \& Almkvist, O. (2003). The assessment of fitness to drive after a stroke: the Nordic Stroke Driver Screening Assessment. Scand J Psychol, 44(1), 23-30.

Lundqvist, A., Alinder, J., Alm, H., Gerdle, B., Levander, S., \& Ronnberg, J. (1997). Neuropsychological aspects of driving after brain lesion: simulator study and on-road driving. Appl Neuropsychol, 4(4), 220-230.

Maycock, G. (1996). The safety of older drivers in the European Union: AA Foundation for Road Safety Research.

McKenna, P., Jefferies, L., Dobson, A., \& Frude, N. (2004). The use of a cognitive battery to predict who will fail an on-road driving test. Br J Clin Psychol, 43(Pt 3), 325-336.

Nouri, F.M., \& Lincoln, N.B. (1992). Validation of a cognitive assessment: predicting driving performance after stroke. Clinical Rehabilitation, 6, 275-281.

Nouri, F.M., \& Lincoln, N.B. (1993). Predicting driving performance after stroke. BMJ, 307(6902), 482-483. 
Odenheimer, G.L., Beaudet, M., Jette, A.M., Albert, M.S., Grande, L., \& Minaker, K.L. (1994). Performance-based driving evaluation of the elderly driver: safety, reliability, and validity. $J$ Gerontol, 49(4), M153-159.

Patomella, A.H., Tham, K., \& Kottorp, A. (2006). P-drive: assessment of driving performance after stroke. J Rehabil Med, 38(5), 273-279.

Pedretti, L.W. (1996). Occupational therapy: practice skills for physical dysfunction: MosbyYear Book, Inc., 863.

Radford, K.A., \& Lincoln, N B. (2004). Concurrent validity of the stroke drivers screening assessment. Arch Phys Med Rehabil, 85(2), 324-328.

Radford, K.A., Lincoln, N.B., \& Murray-Leslie, C. (2004). Validation of the stroke drivers screening assessment for people with traumatic brain injury. Brain Inj, 18(8), 775-786.

Schanke, A.K., \& Sundet, K. (2000). Comprehensive driving assessment: neuropsychological testing and on-road evaluation of brain injured patients. Scand J Psychol, 41(2), 113-121. 